\title{
Psychosocial Hazards faced by Healthcare Workers at a Public Hospital
}

\author{
${ }^{*}$ Tricia Braithwaite' and Shanomae Rose ${ }^{1}$ \\ ${ }^{1}$ Department of Environmental Studies. Faculty of Earth and Environmental Sciences. University of Guyana - \\ Turkeyen Campus. Greater Georgetown, Guyana. *triciafia003@gmail.com.
}

DOI: $10.52377 / C N U F 7812$

Public health institutions are an integral part of Guyana's society, within which healthcare workers play a pivotal role. However, research indicates that Guyana's health facilities are under staffed, which can lead to the risk of on-the-job injury. Moreover, other environmental conditions such as the overcrowding of wards, lack of pharmaceuticals and other institutional deficiencies can lead to stress and result in workplace violence. Thus, the objectives of this research were to determine the psychosocial hazards faced by healthcare workers of a public hospital; to ascertain the contributing factors; and to identify the mechanisms that they employ to cope with psychosocial hazards. Using a descriptive, quantitative study design and cross-sectional method, questionnaires were administered to 207 participating healthcare workers of a public hospital. The results showed that bullying and harassment (34.3\%), violence (34.8\%), work-related stress (70.5\%), lone work (24.2\%), shift work (41.5\%), and heavy workload (71.5\%) are psychosocial hazards faced by the healthcare workers. Analysis of the factors that contributed to psychosocial hazards revealed a positive correlation between lack of experience and workload ( $p$-value=0.015), and being bullied and harassed ( $p$-value=0.009). A positive correlation was also determined between healthcare workers' qualification and high workload (p-value $=0.018)$. Other factors noted were age (25.6\%), gender (19.8\%), attitude (78.3\%), patients' level of comfort (0.5\%), and years of service (0.5\%); which increase the likelihood of hazards experienced. The findings also demonstrated that drinking (7\%), eating (26\%), exercising (22\%), listening to music (5\%), and sleeping (15\%) were the most frequently reported coping mechanisms. This research found that these psychosocial hazards do not usually occur in isolation, and exposure to such hazards may have consequences such as workplace dissatisfaction and staff reduction.

Keywords: Psychosocial hazards; healthcare workers; public hospital 